\title{
Vulval Infection
}

National Cancer Institute

\section{Source}

National Cancer Institute. Vulval Infection. NCI Thesaurus. Code C78716.

An infectious process affecting the vulva. Causative agents include human papillomavirus, herpes virus, Trichomonas, and Candida. Symptoms include itching and pain in the genital area and discharge. 